\title{
Zwischen den Gezeiten
}

\author{
Griechische Wikinger, teuflische Götter, natürliche Gewalt \\ in Lars von Triers Medea (1988)
}

BARBARA BOLLIG

\begin{abstract}
This paper illustrates Lars von Trier's Medea as a character »in-between «: she is both goddess and human, loving and furious, victim and culprit, fascinating and dangerous. Her strongest connection is to the power of the ocean and to water in general-omnipresent, of course, at the coast of Jutland and in the swamp to which she is covertly banished-an element just as vital and fatal as she is to those surrounding her. In her longing for her past life and the homeland she was forced to leave with Jason, to be taken to a particularly Viking Corinth under Creon, she utilizes conflicting character traits to pave a way to emancipation and homecoming as a femme révoltée, fighting suppression and ascriptions of social deviance. Her »existence in-between « is shown as manifested in her appearance, emotions, cultural (un-)belonging, and genealogy, as well as in the dangerous nature ascribed to her by those assessing her actions, particularly the taboo of infanticide.
\end{abstract}

Title: Between the Tides. Greek Vikings, Devilish Gods, and the Power of Nature in Lars von Trier's Medea

Keywords: Lars von Trier (* 1956); myth; gender; film; lieu de mémoire

\section{Mythostransposition: Nordische Medea}

>Nach ihr die Sintflut< ist, so könnte man meinen, das sprichwörtliche Motto von Lars von Triers Film Medea (DNK 1988) - der Adaption des Mythos des Euripides, basierend auf dem verworfenen Drehbuchmanuskript Carl Theodor Dreyers. Meister und Schüler führen, bildgewaltig-sonderbar, die oft bedachte Dichotomie Jason-Medea auf und bedienen sich hierzu eben jener Zuschreibungen, die sich seit der Antike in mannigfaltiger Ausführung im Medea-Stoff manifestiert haben. Wie in vielen seiner späteren Filme zielt Lars von Trier auch mit seiner für das dänische Fernsehen produzierten Medea auf extreme emotionale Reaktionen bei seinen Zuschauer:innen ab und spielt mit jenen Tabus, die Hollywood insbesondere im Umgang mit weiblichen Figuren wenig ausführt und die in seinen Figuren naheliegendes Folgeverhalten auslösen, von Depression über Gewalt bis hin zu Mord. Auch in diesem Film gibt es keine Auf- oder Erlösung für die Rezipient:innen, ist doch auch nach manifestem Aktivismus die Protagonistin in existenzielles Schweigen gehüllt und sichtbar nicht an einem glücklichen Ende angekommen. Mit ihrem Verhalten gestaltet sich die Figur Medea 
(Kirsten Olesen) ebenso gefährlich, mächtig, friedlich wie die sie in von Triers Film umgebende Welt an der Westküste Jütlands, die mit zahlreichen ästhetischen Landschaftsaufnahmen abgebildet wird - doch auch durch ihre Sandbänke, stürmische See und zahlreichen sie spickenden Schiffswracks keineswegs als ungefährliches Gewässer zu bezeichnen ist. In dieser Fremde drückt Medea ihre Sehnsucht nach der Heimat aus, die auch mit einer Sehnsucht nach innerem Frieden und Freiheit von Jason (Udo Kier) einhergeht. Schon ihr Name fungiert als Mythologem, welches nicht nur im Rahmen eines zentraleuropäischen kulturellen Gedächtnisses ein unverwechselbares Narrativ aufruft. In ihrer ruhelosen Transposition in/auf die Nordsee und an die dänische Küste manifestiert sich in Medea ein Zusammenspiel verschiedener idividueller Erinnerungen, nationaler Diskurse und kultureller Verortungen ihrer Person und ihrer Taten gleichermaßen. Ihr letztlicher Sieg (so entbehrungsreich dieser auch ist) wird hier klassisch auch als Sieg der Natur über die kultivierte Welt des Staates bzw. der Männer inszeniert - Medea bleibt hingegen ein intersektionales Subjekt, weder der einen noch der anderen Welt gänzlich zugehörig. Sie ist, in Abwandlung eines Goethe'schen Diktums, >teuflisch menschlich göttlich

Lorraine Daston führt diesbezüglich insbesondere die Sonderstellung Medeas als hybrides Wesen an - besonders in der ihr immanenten Hervorhebung ihrer griechischen Herkunft als Figur eines mythologischen Kanons -, »[she] hovers between the divine and the bestial«, wird von dem ihr abtrünnigen Jason gar als »hateful to gods and human race alike « diffamiert und als wahnsinnig verleugnet - doch »Medea is not mad - on the contrary, she is clever and calculating from beginning to end - and the implication is that she is not merely evil but inhuman, another kind of nature altogether.« (Daston 2002: 381f.) Diese Charakterisierung der Kindsmörderin nimmt bereits Friedrich Nietzsche vor, wenn er hervorhebt, Medea sei in ihrer Rohheit »bis zum Thier ern «i>edrigt«, andererseits »übersteig[e sie] die Grenzen der Menschlichkeit« in die Sphären der Übermenschlichen (Nietzsche, zit. n. Lütkehaus 2001: 205; edit. Ergänzung i.O.). Ihre Andersartigkeit, wider die für Kreon, Jason und alle anderen sie Umgebenden begreifbare Natur, ist der Grund für die ewige Xenophobie, die sich ihr entgegenzuwerfen scheint, wo immer sie auftaucht: am Strand mit Jason, vor den Toren zu Kreons Felsenburg, selbst auf einer Lichtung im Wald, wo Jason sie webend auffindet - er und alle ihr Gegenüberstehenden diskreditieren, fürchten und verteufeln sie. Allein in der Nähe des Meeres bzw. der Sümpfe Jütlands werden feindselige Stimmen erstickt, vor den Grenzen des äußerlich zivilisierten Staates erlangt Medea die Oberhand. Indem von Trier den Mythos an der beinahe unwirtlichen, archaischen Küste Jütlands in einer anhaltenden, windigen Feuchtigkeit aufleben lässt, alludiert er neben römisch-griechischen Mythen auch nordische Narrative von tabuisierter Rache (vgl. Joseph/Johnson 2008: 116) in einem nach Gilles Deleuze und Félix Guattari gleichermaßen glatten wie auch gekerbten Raum (vgl. Deleuze/Guattari 2006). Kreons Gesetze strukturieren diesen Raum eindeutig, Medea hingegen unterwirft sich diesen Strukturen nicht, sodass ihre Positionierung den Raum als von Verwerfungen gezeichnet und, ihrer Eigenverortung außerhalb des nordischen Korinths entsprechend, eine im 
wahrsten Sinne des Wortes fluide Welt entstehen lässt. Für sie ist der eigentlich gekerbte Raum des Staates so glatt, wie es das Meer in seiner Unbezwingbarkeit und Gesetzlosigkeit aus Perspektive der Seefahrenden ist, denen sich Kreon qua Eigencharakterisierung zuschreibt. Dies, wie auch die Praktik der Synchronisation der Figurenrede und die Disparität zwischen Bildern und Ton, Rückprojektion und hartem Schnitt, trägt genauso zu Entgrenzungen bei wie die Aufhebung von Dichotomien wie die von Kultur und Natur, männlich* und weiblich ${ }^{* 1}$ (vgl. auch Müller 2000: 14). Dem Ton des Films, der natürlichen Geräuschkulisse, ist eine bedeutungsentscheidende Rolle beizumessen, sie ermöglicht einen »wordless exchange that reflects emotional and psychological states. This is exemplified in the Prologue in which the natural sounds slowly become quieter as we listen to the lapping of waves, a single birdcall, and, ultimately, Medea's loud gasp for air as she emerges from the sea.« (Joseph/Johnson 2008: 116)

Diese Geburt aus dem Wasser ist es, die gleichzeitig auch das Todesurteil derer besiegelt, die Medea entgegenstehen; von Triers Film manifestiert die Juxtaposition von Leben und Tod, Liebe und Rache, Niedergang und Aufstieg in der natürlichen Opposition zwischen den Elementen Erde - zuzuschreiben der Felsenburg, Platzhalterin für Korinth (auch wenn diese Stadt auf eine erfolgreiche Geschichte des Seehandels zurückblickt), und mit ihr der Statthalterfigur Jason - und Wasser, aus dem sich Medea erhebt, mit neuer Kraft und Macht eine Verbindung, die bereits im antiken Text des Euripides prominent wird (vgl. ebd.: 131). Wasser (und Wellen) sind durchgehend weiblich konnotiert und kontrolliert. Sie untermalen von Beginn an die bewusste Entscheidung Medeas, sich an Jason zu rächen, anstatt sich von der Farce zu Hofe ertränken zu lassen, verunsichern die Männer, die ins Wasserreich einzudringen wagen, und fordern schließlich Jason bildgewaltig als Ertrinkungsopfer im roten Wellenmeer der Gräser. Mit seiner Umsetzung eines »zeitlose[n] Schicksalsdrama[s]« (Björkman 2001: 120), dessen Grundkonflikt sich in der Installation der Bilder äußert, findet sich von Trier epochal in eine späte Phase des Postmodernismus eingeordnet, in der, so Marion Müller, eine eifrige Interaktion von Ethik und Ästhetik stattfindet und die Rezipierenden dazu anhält, das Gesehene unter ethischen Gesichtspunkten zu bewerten, statt lediglich die ästhetische Ausgestaltung einzubeziehen (vgl. Müller 2000: 15). In seiner Zusammenarbeit mit Tom Elling und Tómas Gislason bei der bildlichen Umsetzung der symbolhaften Einstellungen des Films und der Aufladung mit Niels Vørselns Vorstellung der »Gefährlichkeit der Natur« (Björkman 2001: 44) - die hier von Medea als Zwischenwesen und damit aus der korinthischen Zivilisation Ausgeschlossene vertreten

1 Die Asterisken verweisen auf die Auflösung der als binär trennbar angesehenen Geschlechterdichotomie und die seitens der Autorin bevorzugte Ansicht eines Spektrums an Geschlechtszugehörigkeiten, die vom Individuum selbstständig und immer neu gewählt werden können. Wenn im Folgenden auf 'männlichı oder 'weiblich، rekurriert wird, geschieht dies in Anlehnung an eine antiquierte Auffassung von äußeren Attributionen der Kleidung, nicht des biologischen Körpers als solchem, als eher stereotyp männlich oder eher stereotyp weiblich. 
wird -, findet eben jene schauerliche Schönheit Einzug in Medeas Abrechnung. Doch ist sie nicht die kalte, emotionslose Rächerin, auch nicht die affektierte Wahnsinnige, als die sie teils dargestellt wird; vielmehr ist sie Erduldende, Leidende und doch Entschlossene, Schöpferin von Leben und Zerfall gleichermaßen - und damit eine Frau, die auch die ihr oktroyierte bürgerliche ${ }^{2}$ Geschlechterkategorie aufbricht.

Die Hauptfigur ist Gefahr und Faszinosum zugleich, auf eben jene Art und Weise, wie auch das Meer mit sanftem Rauschen und unergründlichen Tiefen diesen Doppelcharakter innehat. Wo Medea in den ersten Einstellungen des Films von der einlaufenden Flut erst um-, dann überspült und schließlich verschlungen wird, nur um wenig später wieder aufzutauchen und sich im weiteren Verlauf das Meer in seinen mannigfaltigen Formen untertan zu machen, bis sie schließlich darauf entgleitet, geht der Rest der Figuren unter - sprichwörtlich wie real, zerschellend an Medeas Rage. »The idea [...] was for her to hold her breath as the water rose. [...] I imagined that she was holding in all her anger and rage by holding her breath. That enormous rage she feels and carries within her « (Leonard 2015: 2), erklärt von Trier sein Prélude, in welcher der Perspektivwechsel von Top-Shot zu Normalsicht die Rezipient:innen strudelartig in die Welt der Medea hineinzieht und diese auf Augenhöhe erlebbar macht.

\section{Kulturelle Mnemotopol: Weiblichkeit und Meer}

Das Meer ist hier jedoch nicht nur das Elementare, das Medea neuerlich gebiert und bekräftigt, vielmehr stellt es auch einen Erinnerungsraum dar. Zusammen mit den anderen Feuchtgebieten steht es zirkulär für eine Entfremdung der Frau von Heimat und Natur ${ }^{3}$ unter Jasons Anleitung, die Rückbesinnung auf

2। Lars von Triers Medea durchbricht eine klassisch bürgerliche Markierung des öffentlichen Raumes als männlich, als durch die Männer von Korinth dominiert und jeglicher weiblichen Figur absent, indem sie sich Schwellenräume zu eigen macht. Im öffentlichen Raum, in dem sie allein Objektstatus hat und in Abhängigkeit von männlichen Subjekten existiert, hat sie als Fremde, Mächtige und Emanzipierte keinen Seinsort; allein im scheinbar unkultivierten, also männlich unbezwungenen Schwellengebiet des Sumpfes wird sie stärker zu einem eigenständigen Subjekt. Jedoch bleibt sie - in den Augen ihrer Widersacher - unvollkommen, ein Abjekt (s.u.), da sie zwar gegen bestehende Normen (bspw. bürgerliche Rollen, Geschlechterbilder, Handlungsabläufe) verstößt und eigenständig agiert, jedoch im korinthischen Gebiet nicht Fuß fassen kann und wird, nachdem sie mit ihren Übeltätern abgerechnet hat.

3 | Ikonographisch wird durch die wiederholte Darstellung Medeas außerhalb der königlichen Felsenburg augenscheinlich eine tradierte und klischeehafte Verbundenheit zwischen Frau und Natur evoziert. Jedoch gestaltet sich der Bezug bei eingehender Analyse mehrdeutig. So handelt es sich bspw. nicht um eine wilde, ursprüngliche Natur, die sich außerhalb der Menschenstätte ausbreitet, sondern um eine kultivierte Natur, die die Korinther in ihren rituellen (vgl. Fackelzug und Hochzeitsnacht) wie öko- 
das Zurückgelassene sowie schließlich die Rückkehr des veränderten, erschütterten, aber eben auch bereinigten Selbst in eine Heimat. Medea wird bildlich überschwemmt von der Untreue Jasons, beschließt nach Atem ringend ihr weiteres Vorgehen, sammelt Kräfte (und Gifte) im Sumpf und erhebt sich schließlich über alles, was sie zu ertränken drohte. Wenn sie eines nicht ist, dann konform zu all dem, was Kreon proklamiert; sie wird entsprechend nicht im Inneren seiner Machtzirkel angesiedelt, sondern »in a world where animistic magic is being relegated to the margins by mercantile and political society: [...] the murky bogland and seashore of the North « (Christie 2000: 155). Sie ist eine Figur des Liminalen, die mit der Natur mise en scène interagiert, gleichberechtigt und auf beinahe magische Weise. Die Kultur der Korinther, der korinthischen Wikinger in ihren nordischen Lagern vielmehr, bleibt ihr fremd und sie, aus Perspektive eben jener, als >Abjekt ${ }^{4}$ in ihrer Zwischenwelt gefangen und doch heimatlos wie ein Fisch.

Viel bedeutender ist jedoch, dass Medea selbst in ihrer Präsenz physischer und evokativer Art einen Gedächtnisraum, eine Projektionsfläche und einen Resonanzraum darstellt, die von Trier mit zahlreichen kulturspezifischen Symboliken auflädt und sie so zur Manifestation eines transkulturellen Gedächtnisses macht. Durch die Anführung des Mythologems Medea als Figurenname ist die Hauptfigur der informierten Rezipierendenschaft als eindeutig in der klassischen griechisch-römischen Mythologie und einem daraus über Jahrtausende

nomischen (Seefahrernation, Umschlagplatz für Waren/Menschen) Handlungen einbetten und entsprechend kultivieren. Selbst Medeas Produktivität in der wilden Natur des Sumpfes durchbricht das oben genannte Klischee; sie bedient sich zwar der vorhandenen Schnecken und Pflanzen für ihre todbringende Giftmischerei, jedoch ist auch hier eine naturbezogene Ökonomie nachzuvollziehen, die nicht mit dem klassischen Bild der in der Schönheit der Natur passiv existierenden Frauenfigur zu vereinbaren ist.

4 | In ihrem Langessay Powers of Horror (1982) geht Julia Kristeva sowohl auf den Prozess der Abjektion als auch auf das Abjektwerden/-sein des Individuums ein. "The abject has only one quality of the object - that of being opposed to it." (Kristeva 1982: 1) Diese Gegensätzlichkeit macht also das Abjekt als solches aus - das sich entgegen der bestehenden Norm verhaltende Wesen, das sich auf eine quest of desire begibt, seinen eigenen Gefühlen und Trieben folgt und sich damit von der Norm abjektiviert. Abjektion wird hier durch all das hervorgerufen, was die bereits etablierte Identität, das gefestigte System, und/oder die bestehende Ordnung stört. Hinzu kommt jedoch auch die Motivation des Subjekts, sich mit etwas extrinsisch Vorhandenem zu identifizieren - schlägt dieser Versuch fehl, ist dies darauf zurückzuführen, dass das Subjekt sich innerlich, im übertragenen Sinne auch ideell, dazu nicht in der Lage sieht. Die Unmöglichkeit des Zugehörigkeitsempfindens konstituiert das Sein des Subjekts und macht es zum Abjekt. Medea wird von ihren männlichen Gegenspielern abjektiviert, indem diese inr den Eintritt und die Situierung in dem ihr fremden System der korinthischen Felsenburg verwehren - zugleich findet sich die Zauberin durch die kulturelle wie spatiale Transposition in die fremden nordischen Gefilde ihrer Heimat entwurzelt. Ihre Subjektwerdung in der neuen Umwelt stagniert. 
erwachsenen Diskurs und in diversen Kulturen als weltliterarisches Sujet etabliert erkenntlich. In Diskurs und Sujet manifestieren sich einerseits kulturspezifische Normen und Werte, andererseits Universalien einer menschlichen Verfassung, die alleingenommen singulär kultur- oder nationalspezifisch gelesen werden können, sich zugleich aber gerade über einzelne Kulturkreise hinaus als produktiv und bedeutungskonstitutiv erweisen und somit zu einer transkulturellen Interpretation einladen. Durch die Juxtaposition der vorzeitlich-mediterranen Mythosfigur und des ebenfalls zeitentbundenen skandinavischen Ortes unternimmt von Trier nicht nur eine szenische Untermalung der medeischen Gefühlswelt durch stürmisches Nordmeer, er stellt auch den Universalcharakter der sich entfaltenden Tragödie aus und rahmt diesen visuell mit aufgepeitschter See, unheilheischenden Strömungen und angsteinflößendem Wellenrauschen bei gleichzeitigem menschlichen Schweigen. Medea vereint diverse Mythologien um Seefahrt, Mütterlichkeit und meerhafte Mystik in sich, die nach Jan Assmann prägend für ein aktives und präserviertes kulturelles Gedächtnis einer Gemeinschaft sind und den Traditions- und Werteapparat von Kulturen nachhaltig prägen (vgl. Assmann 1988). Medea als tragisch-literarische Figur stellt nicht nur einen Teil des in Schriftzeugnissen konservierten kulturellen Kanons dar, also einen aktiven Teil von Literatur und Mythos als manifestem Gedächtnis. Verarbeitungen ihrer Geschichte und Arbeiten am Mythos Medea, wie sie hier in Form des Filmes vorliegt, markieren mitunter gleichermaßen eine aktive Gedächtnisarbeit an Literatur und Kultur.

Trotz der ihrem Namen anhaftenden kulturellen Zuschreibungen ist Medea in ihrer untrennbaren Verbindung mit Meer und Zwischenräumen im Film weiterhin ein heimatloses Abjekt ohne eindeutige kulturelle Verwurzelung auch im antiken Mythos ist sie in ihrer eigentlichen Heimat Kolchis nicht mehr willkommen, kann in Korinth als Barbarin nicht Fuß fassen und hat allein die Aussicht, in Athen Asyl zu suchen; ihre Heimat ist Jason, dem sie sich, je nach Version des Mythos, durch Mimikry auch kulturell anzugleichen sucht, freilich ohne Erfolg. Ebenso wie der wässrige, konstant von Wellen geprägte Raum, in dem sie sich bewegt und den sie für andere ungangbar macht, ist ihr Dasein geprägt von der Unmöglichkeit einer Unterwerfung ihrer Macht. Wie das Meer, aus dem sie zu Beginn des Films entspringt, wird sie genutzt, wird zum Medium der Erfüllung einer machtgeprägten Agenda; Wikinger und Argonauten bewegen sich über den Nichtort Meer zur Kolonialisierung der umliegenden Welt respektive zur Abenteuerfahrt, das Meer trägt und ernährt sie, Heimat ist es dennoch nicht - Medea verhilft Jason, dem Mythos entsprechend, zu Vlies und Macht, gebiert ihm Nachkommen und wird doch letztlich und gegen eine neue Möglichkeit der Verwurzelung im skandinavischen Korinth ausgetauscht. Wie das Meer ist sie für den Menschen/den Mann Jason ein temporärer Seinsraum, dem emotive Werte und Erinnerungen abgesprochen werden, jedoch auch ein lieu de mémoire nach Pierre Nora (vgl. Borschberg/North 2010). Von Trier evoziert durch die Juxtaposition filmischer Aufnahmen als visuelle Gedächtniselemente und Medea als Figur eines literarisch-kulturellen Gedächtnisses die Notwendigkeit, beide Komponenten nicht nur in ihrer individuellen Symbolik zu 
lesen. Sie sind auch in ihren symbolischen Wechselwirkungen zu betrachten und anzuerkennen - ein Prozess, den der Film anstößt, der allerdings durch die individuelle, kulturell geprägte und subjektive Diskursbildung vor dem Hintergrund gelesener medialer Texte erst durch die Rezipient:innen ausgeführt wird. In diesem Lektüreprozess findet eine Vermischung individueller und kulturspezifischer Komponenten statt, die Medea, wie auch das ihr dominant beigeordnete Meer, als lieux de mémoire divisés (vgl. ebd.) etablieren, als Räume kultureller, spatialer und historisch-mythischer Intersektionalität.

Allein die Gedächtnisleistung der Rezipierenden und die interpretative Bezugsetzung diverser kultureller Spezifika zueinander konstituieren Medea als eine Art menschlichen kulturellen middle ground, der verschiedene Komponenten einzelner kultureller Räume zu einem produktiven Austausch zusammenkommen lässt und die Filmfigur zu einer Echokammer transkultureller Symboliken macht, zu denen offenkundig auch das Meer in seiner Ambivalenz als Element der von Trier'schen Medea gezählt werden muss. Es ist ein Raum ohne geltende menschliche Gesetze und doch ein von (Natur-)Gewalt bestimmter, es spendet Leben und ist in der Lage, es zu nehmen (nurture vs. torture) und gilt gemeinhin als männlich dominiert und damit weiblich konnotiert und objektifiziert, wenn auch, besonders in der Antike, der Ozean durch männliche Gottheiten wie Poseidon stark männlich gelesen wurde. Ihm entsprangen jedoch zahlreiche weibliche Kinder - als Wellen, wie die Snorra- und Lieder-Edda des 13. Jahrhunderts ausführen, Nixen und Sirenen (vgl. Helmreich 2017). Die weibliche Codierung des Meeres stellt eine spätestens seit der Entstehung der bürgerlichen Gesellschaft kulturübergreifende Konstante dar, wie sie Stefan Helmreich am Beispiel der Wellen herausstellt, die auch in von Triers Film hochgradig symbolträchtig sind. Er verweist auf Klaus Theweleits Männerphantasien (1977/78), der die wiederkehrenden Topoi der Frau im/als Wasser, als reißender Strom und stürmische See, als grenzenloser, verlockender, gefährlicher Körper, besonders aber die Vagina als schäumend und parallel zum auf Wellen getragenen Seeschaum liest (vgl. Helmreich 2017: 31). Ergänzend hierzu führt Helmreich Luce Irigarays historisch-kritische Forschung an, die unterstreicht, dass »historically, the properties of fluids have been abandoned to the feminine (ebd.). Damit bewegt sich Helmreich am Puls der Zeit des Anthropocene Feminism, durch Stacy Alaimo geprägt, der an der Intersektion zu Critical Race Theories davon ausgeht, dass materielle Einheiten - wie Wellen sie auch sind - durch Geschlechterrhetoriken geprägt (vgl. ebd.: 30) und damit mit individualkultureller Symbolik aufgeladen und entsprechend im Prozess einer rezipierenden Bedeutungskonstitution als Manifestationen eines kulturellen Gedächtnisses aufzuschlüsseln sind. Medea werden eben jene Charakteristika zugeeignet, sie stellt ebenfalls eine faszinierende und gefährliche Macht dar, ist durch ihre direkte Nähe zum Meer, aus dem sie entsteigt, untrennbar mit ihm und seiner Symbolik verbunden und herrscht über die Wellen, die Jason verschlingen und über die sie dem skandinavischen Korinth entflieht. Die sich hier manifestierende Monstrosität stellt zudem ein traditionelles Element aller Diskurse dar, die sich um den Medea-Mythos ranken; eine symbolische Verwandtschaft ist nicht zu leugnen. 
Selbst in der konnotativen Ambivalenz der Wellen als weiblich in der altnordischen Mythologie gegenüber einer ihnen attestierten Männlichkeit in der griechischen Mythologie (vgl. ebd.: 33-35) bleibt von Triers Medea sichtbar, stellt sie doch ein geschlechterperformativ androgynes Wesen dar.

\section{ZWISCHEN DEN GEZEITEN: Heterotopie als Widerstandsort}

Durch die gemeinsamen Kinder ist Medea an Jason und damit an die Erde gebunden und erscheint erstarrt in ihrem Dasein. Erst die Zerschlagung dessen, was sie bindet, ermöglicht eine Loslösung, durch die Nähe Medeas zum Wasser entsteht, so Andrea Schorr (vgl. 2009: 23), gar die Möglichkeit einer Reinigung von Schuld, mit der die Kolcherin sich belädt. Am Ende scheint eben dies einzutreten: Medea bewegt sich auf dem Seeweg fort von der Stätte des Infantizids, ihr Haar weht frei im Wind vor einem blauen Himmel, ebenso weit wie das Meer, während in einer Parallelmontage Jason in einem Gräsermeer versinkt, das filmisch aus Medeas Locken hervorgeht. Er geht zugrunde auf der Erde, während sie sich freimachen kann von irdischen Fesseln; dea ex machina entschwebt sie nicht, wie ihr antikes Vorbild, auf einem Schlangenwagen in luftige Höhen, sondern gleitet auf Wellen getragen einer irdischen Existenz entgegen. Sie gibt sich dem zurück, aus dem sie sich zu Beginn erhoben hat: dem Meer; in einer äußerlichen Ruhe, die dem anfänglichen Überschlagen der Bilder kontrapunktisch gegenübersteht. Insbesondere durch das schneller werdende Rotieren der Kamera in Draufsicht auf die im Watt liegende Frau wird »die aus den Fugen geratene Welt ohne Orientierungspunkte, die den Seelenzustand Medeas widerspiegelt«, evoziert - es »drängt sich außerdem die näher liegende Assoziation eines Wasserstrudels auf, eines Malstrøms, der auf der Formebene den Strudel der Ereignisse vorwegnimmt, von dem Medea mitgerissen wird.« (Müller 2000: 166) Diesen Ereignisstrudel bedingt sie jedoch auch mit, und er droht sie ähnlich einer Sünden- oder Sintflut zu überschwemmen. Diese immanente Drohung, eine wiederkehrende Warnung vor dem sich Anbahnenden, korrespondiert mit den Stimmen des in der klassischen Tragödie vorhandenen Chores, der hier nicht figurativ in Erscheinung tritt, sondern in der Natur vorhanden ist. »Die Kommentare des Chores müssen also in den Bildern liegen.« (von Trier, zit. n. ebd.: 163) So ist es möglich, die letzte Zusammenkunft Medeas und Jasons am Strand, vor den Morden, in ihrer stilistischen Umsetzung als Chorkommentar in einer Art Zukunftsvision zu deuten. Hier finden sich die dem Paar zugeschriebenen Elemente vertauscht, wann immer Jason gezeigt wird, ist der Hintergrund ausgefüllt von Meer und schäumenden Wellen, während Medea von Dünen eingerahmt wird. Die Farbcodierung dieser Aufnahmen hingegen wird durch einen Blue Box-Effekt vertauscht; so ist das Meer hinter Jason grün, die Dünen um Medea hingegen sind blau gehalten, was, wie auch Müller (vgl. ebd.: 16o) herausstellt, auf die letzten Aufnahmen des Filmes hindeutet, in denen die Figuren sich wieder >in ihrem Element $<$ befinden. Auch 
die einrollenden Wellen hinter Jason deuten auf dessen letztendlichen Untergang in Medeas Tun hin.

Wie bereits angedeutet, spielt jedoch nicht nur das Meer in seiner Urform eine bedeutende Rolle: Vor allem dem Sumpf, in dem Medea die Zutaten für ihr Gift zusammenklaubt und in dem Kreon, seiner Überlegenheit beraubt, Medea folgend im Nebelmeer zu verschwinden droht, ist besondere Bedeutung beizumessen. Als Heimatlose in den klar definierten Umgebungen Korinths findet Medea erst im Heterotop, dem eigentlich unbewohnten Niemandsland des Moores, einen Seinsort. Doch auch hier kann die Giftmischerin sich ihres Elementes bedienen: Als Zwischenform von Wasser und Land respektive Wasser und Luft sind Medea sowohl Sumpf als auch Nebel nützlich und untertan. Sie ermöglichen ihr ein furchtloses Verbleiben, indem sie Kreon und seine Männer verängstigen und ihnen dort sicheres Geleit und Orientierung nehmen, wo Medea sich ihrer Früchte für ihre grausame, doch gleichzeitig auch emanzipatorische Rache an Jason bedienen kann. Diese divergierenden Wahrnehmungen der Heterotopie manifestieren sich auch in allgemeineren Rezeptionen derselben. Rodney James Giblett stellt heraus, dass Sümpfe

have been seen by many in the west as places of darkness, disease and death, horror and the uncanny, melancholy and monstrous - in short, as black waters. Yet a minority in the west and the indigenes of Australia (and no doubt elsewhere) regard wetlands as places of both life and death, light and dark, as biologically rich and fertile, [...] vital for life in earth. (Giblett, zit. n. Müller 2000: 168)

Müller fügt hinzu: »[S]chon immer wurde mit dem Sumpf das Weibliche assoziiert (Schleim, Gebärmutter). Er gilt als [...] Zufluchtsort für Flüchtlinge oder Widerstandsbastion und Versteck für Anführer oder allgemein als Ort, wo sich >the Other < aufhält.« (Ebd.) Und dies alles ist Medea: Geflüchtete vor dem Bann Kreons und dem doch unausweichlichen Ende ihrer Elternschaft, Widerständige gegen eine aufoktroyierte Machtkonstellation und Anführerin ihrer eigenen Rebellion gegen Jason, Korinth, Illoyalität und Missbrauch.

\section{Abjektivität in Person und Ort}

Das >Wikingertum $<$ der von Trier'schen Griechen stellt formell eine Assonanz mit dem Seefahrermythos der Argonautica dar, Hinweise auf die Seemacht Kreons und Jasons durchziehen den Film; selbst die endliche Enthebung Medeas von jeglicher irdischer Rechenschaft für ihre Taten dea ex machina auf dem zu Aigeus' Schiff umgeformten Schlangenwagen unterstreicht den Fokus auf die See als dramatis persona. Gar ließe sich darüber debattieren, inwiefern Jason hier als ein dem populären Bild entsprechend frauenraubender Wikinger gelesen werden kann, lässt von Trier doch die Vorgeschichte um dessen Zusammenkunft mit Medea bis auf wenige, an eine Stummfilmtradition erinnernde Bildtafeln weitestgehend im Dunkeln und überlässt sie damit assoziativ der Rezipient:innen, 
während er kritisiert: »Ich bin mit dem Film nicht sonderlich zufrieden. Ich glaube, es liegt daran, daß ich diesen ganzen Wikingerquatsch nicht in den Griff bekommen habe. Egal, wie man solche Sachen umsetzt, gerät das Ergebnis unfreiwillig zu einer Art Maskerade.« (von Trier, zit. n. Björkman 2001: 120) Wer sich bspw. an Grillparzers Goldenes Vließ (1821) erinnert, mag Medeas blutigen Weggang aus der Heimat als von (blinder) Liebe bestimmt deuten, andere Versionen wie bspw. Dea Lohers Manhattan Medea (1999) stellen existenziellere Fragen nach dem Überleben in Heimat und/oder Fremde jeweils in den Vordergrund. Was diesen >Arbeiten am Mythos ${ }^{5}$ (H. Blumenberg) gemein ist, ist die omnipräsente Verhandlung von Xenophobie, Alienation, Heimatlosigkeit - sowohl im geographischen wie auch im genealogischen Sinne. So ist Medeas

failure to fit in [...] ascribed to purely social causes: she is a barbarian among Greeks. This element is also present in the ancient accounts, but it simply reinforces the root difficulty of Medea's pied nature, part-god, part-mortal, part-beast; heroic male and maternal female. Within the moral order of specific natures, this is the essence of her tragedy and why her deliberations, which stage the conflict among her several natures, are of greater moral import than her deed. (Daston 2002: 392)

5 | Ohne an dieser Stelle erschöpfend auf alle Medea-Texte eingehen zu können, soll dennoch kurz auf den wohl bekanntesten Medea-Film von Pier Paolo Pasolini (Medea, Italien 1969) verwiesen werden. Als gleichermaßen bild- und audiogewaltiges Kunstwerk stellt auch Pasolini seine Medea in Verbindung mit dem Meer dar - jedoch zeichnet sich sein Text visuell gerade durch die Absenz dessen aus. Medea moniert, in Korinth, auf trockenem, sichtbar dürrem Boden, habe sie jede Verbindung zu den sie umgebenden Mächten verloren, ihren Ahnen wie auch den Kräften der Natur. Pasolini reiht sich in dieser Hinsicht stärker in die oben genannte Tradition einer Verbindung zwischen Frau und Natur ein, jedoch wird auch hier durch die Macht der Figur Medea eine einfache Lesart verhindert. Neben zahlreichen Unterschieden weisen die Filme Pasolinis und von Triers einige Parallelen auf, die sich auf das Gros der Mythosversionen übertragen lässt. Beide stellen nicht nur die Figur Medea, sondern auch ihre Perspektive auf das sich entfaltende Geschehen in den Vordergrund; in beiden wird ihre Übermenschlichkeit dargestellt, wenn auch von Trier von einer direkten Anrufung Medeas an ihre Gottheiten absieht; zuletzt - und besonders für den vorliegenden Beitrag interessant - stellt die immerwährende Verbundenheit Medeas mit dem Meer eine Universalie dar. Als Tochter Kolchis' entstammt sie der Schwarzmeerküste, das Meer trägt sie auf der Argo gen Korinth und die besonders in den neueren Versionen des Mythos thematisierte Flucht nach vollbrachten Taten nach Athen geschieht mit Aigeus' Schiff. Für den Medea-Mythos als Teil eines kulturellen Gedächtnisses lässt sich hieraus ableiten, dass, egal ob nun von der von Trier'schen nordischen Medea oder der mediterranen Medea Pasolinis ausgegangen wird, die Betonung der Seefahrt und die ökonomische wie mystische Signifikanz des Meeres für den Mythos und die menschliche Realgeschichte gleichermaßen bedeutungsvoll sind - was die Funktion des Mythos als kulturelles Gedächtnis und die Signifikanz des Meeres (ob als Geschehensort, beinahe eigenständige Figur oder gar schmerzliche Leerstelle) als Mnemotopos, als lebendigen Erinnerungsort, verstärkt. 
Besonders Medeas Dasein als äußerlich meist maskulinisiert markierte Frau stellt sie im filmischen Medium als andersartig heraus, denn obschon sie Kreusa mit Gift zu Leibe rückt und den Kindern Schlingen um die Hälse knüpft, was beides gemeinhin weiblich markiert ist (vgl. Leonard 2015: 5), ist sie doch mit Ausnahme der Schlussszene beinahe sent-körperlicht<. »We never see her body; it is covered by long, tight-fitting black costume, almost like the skin of a sea mammal, suggesting once again her affinity with water and perhaps pointing to Nordic allusions of the mythical selkie (creatures that can transform themselves from seals to humans).« (Joseph/Johnson 2008: 121) Asbjørn Jøn (vgl. 1998: 96-98) stellt das Selkie als eine Mischform aus Mensch und Robbe heraus und verortet es in einer keltischen und nordischen Mythologie; es sei gutmütig und positiv konnotiert; weibliche Selkies könnten in ihren menschlichen Körpern gefangen bleiben, wenn man sich ihrer wie eines Mantels aus abgelegter Robbenhaut bemächtigte, was sie zu einem Leben unter Menschen verdamme, bis sie ihre Haut wieder überstreifen könnten. Obschon hier konkret das Selkie als Referenzobjekt angedeutet wird, liegt insbesondere durch das Verbergen ihrer roten Locken unter einer wie mit Schuppen besetzten Kappe, die die Ähnlichkeit mit einem ergonomisch geformten Tierkopf weiter untermalt, auch eine mögliche Verbindung Medeas zur Symbolik der (See-)Schlange nahe. Diese durchdringt den Medea-Mythos insbesondere in der Version Senecas (um 56 n.Chr.), sei es in Form des bereits erwähnten Schlangenwagens, aufgrund der um den Hals gelegten Schlangen beim Beschwören der Geister der Unterwelt, denen sich Medea überstellt, oder aber durch die Lesart ihrer dramatis persona als Schlange. In ihrem furiosen Dasein wird Medea selbst den Tieren ähnlich: Sie ist intelligent wie eine Schlange und vereint in sich die Möglichkeit, andere zu töten, ohne dabei selbst fatalen Schaden zu nehmen , und zugleich als Mutter und Zauberin Leben zu spenden - zuletzt auch sich selbst. Für Senecas Athener und ihre eigenen Kinder wird Medea eine Schlange im negativsten Sinne, eine giftige Furie, zum schlimmsten Feind von Leben und Ordnung. Im Gegensatz dazu jedoch steht der Aufstieg Medeas aus der Welt. In einem von geflügelten Schlangen gezogenen Wagen wird sie gen Helios gehoben, die vorher tödlichen Tiere ermöglichen ihrer Herrin die Flucht. Poetisch gesehen rahmt Seneca seine Tragödie mit dem Bild der schlangenhaften Furie ein. Mit gespaltener Zunge fragt Medea zu Beginn, wem sie zuerst den Tod bringen soll, bevor sie am Ende, nach begangener Tat, blutbefleckt und Athen vergiftet zurücklassend, in ein anderes Leben entschwebt - Elemente, die auch von Trier aufgreift.

Weiterhin ließe sich die Schuppenstruktur der Kappe auch als fischähnlich ausdeuten, was wiederum die Zuschreibung Medeas zum Wasser untermauern würde, gar ähnlich einer Meerjungfrau oder -hexe (vgl. Müller 2000: 165), die, je nach kulturellem Referenzrahmen, ebenfalls, wie auch Medea, Leben gebend und Leben nehmend gedeutet werden (vgl. Asbørn Jøn 1998: 96; Eason 2008: 149). Medea bemächtigt sich in ihrer schwarz-glänzenden Tracht, einer grundsätzlich in kalten Farben gehaltenen Umgebung und herben, beinahe wettergegerbten Zügen einer Art verbittertem Stolz und geht schließlich, bis auf wenige Ausnahmen, mit einer besonderen Verdrossenheit und Härte gegen ihre 
männlichen Gegner vor (zwar ist auch Glauke zur Gruppe der ihr Entgegenstehenden zu zählen, jedoch zeichnet sie sich durch nahezu gehorsame Passivität aus, indem sie nicht direkt gegen die Rivalin vorgeht, sondern Druck auf Jason ausübt). Die Kolcherin verkörpert die Rolle der femme révoltée, die ihr Schicksal an sich nimmt, in ihrem Aktivismus jedoch »zum Mann werden [muss], um gegen Jason zu kämpfen und den Mord an den Kindern auszuführen. Als die Amme sie fragt, ob sie lieber ein Mann wäre, antwortet sie ohne Zögern mit ja, nachdem sie vorher über die Stellung der Frau in der Gesellschaft geklagt hat.« (Müller 2000: 162) Medeas Androgynie und die Demarkierung ihres Körpers als weiblich* führen visuell zur Aufbrechung eines Genderbinarismus, wenngleich der Film letztlich mit der symbolischen Entfesselung ihrer Haare und der Rückkehr zu semiotischer Weiblichkeit in einer tradierten Gendermatrix verbleibt. Von Medea geht die alles überspülende Macht im Film aus, die benötigt wird, um den existenziellen Konflikt von Stärke, Emotion, Aufopferung und Emanzipation auszutragen, der das Leben der Mutter besonders vereinnahmt. Mit ihrer Rache an Jason, der die Kinder zum Opfer fallen, demonstriert Medea eine besonders große Leidensfähigkeit, sie geht entschlossen und ruhig vor, wenn sie auch kurz hadert, als die Kinder nacheinander am Galgen hängen, und durchbricht so ein proklamiertes Bild ihrer Selbst als kaltblütige Rächerin. Sie ist es, die durch die Tilgung der Söhne vor allem Jasons Fortbestand zerstört, ist dieser doch letztlich abhängig von der Frau, die ihm Kinder gebiert - ebenso wie Kreon, der seine Stammhalterschaft durch den Tod Glaukes ebenfalls ausgelöscht sieht, und Aigeus, dessen Kinderlosigkeit ihn erst in die Gewässer bringt, in denen er auch Medea trifft, die ihm bei der Sicherung seines Erbes behilflich sein will, so er ihr die Abreise aus Korinth sichert. Sie nimmt den Kindern das Leben und befreit sich, was bildlich sonst Jason zukommt, obschon seine Freiheit eine trügerische ist. Er ist

zwar in der freien Natur zugange, doch auch dort ist er gefangen. Zum einen sieht man inn [während der Verfolgung Medeas und der Kinder; B.B.] inmitten der Baumstämme, aus denen er keinen Fluchtweg findet, wie oft er auch hin- und herreiten mag. Zum anderen wird er durch den Kader der Kamera im Grasmeer festgehalten und kann aus dessen Grenzen nicht ausbrechen. (Ebd.: 167)

Doch auch Medea ist stetig unfrei und erst

nach vollbrachter Rache und nachdem sie auf Aigeus' Schiff in Sicherheit ist, darf die androgyne Medea wieder zur Frau werden und ihr Haarnetz abnehmen, so dass inr rotbraunes Haar im Wind wehen kann. [...] Sie ist nur eine relative Siegerin, denn sie hat alles verloren, auch ihren Status als Mutter und so einen wichtigen Teil ihrer Weiblichkeit, die sie nun zumindest äußerlich wieder annimmt. (Ebd.: 162) ${ }^{6}$

6 | Es sei an dieser Stelle auf die Problematik einer Zuschreibung von Weiblichkeit aufgrund biologischer Mutterschaft verwiesen, die zwischen sozialer Rolle, Genderperformanz und biologischem Geschlecht nicht ausreichend unterscheidet. 
Auf indiskutabel grausame Weise entledigt sich Medea der ihr durch Jason aufgebürdeten Opferrolle in ewiger Heimatlosigkeit ohne jegliche kulturerelle Zugehörigkeit und ihre Kinder; so verweigert sie sich der Erfüllung patriarchaler Machtphantasien, indem sie als Schicksalsgöttin eine Unglücksflut über diejenigen heraufbeschwört, die sie fesselten. In ihrem gelösten Haar sehen Susan Joseph und Marguerite Johnson nun nicht länger die Androgyne, sondern stellen fest, Medea sei »once again a nymph, a play on the multiple meanings of nymph as unmarried woman, water creature, and mournful presence« (Joseph/Johnson 2008: 123).

\section{EMOTIO OHNE AFFEKT}

Ähnlich wie die Titelfigur in Heinrich Leopold Wagners Drama Die Kindermörderin (1776) zeichnet sich auch von Triers Medea durch emotionale Janusköpfigkeit aus: Bestimmt vergiftet sie die Zacken der Krone Glaukes und flieht danach mit den Söhnen - Kalkül und Hass dominieren diese Taten; erreicht sie jedoch den Galgenbaum, lässt letzte Fürsorge für die Kinder sie bis zuletzt stocken. Auch nach dem Tode der Söhne bleibt ein Schleier der Grausamkeit ihrer Tat an ihr haften, der sich auch durch die sinnbildliche Befreiung der roten Mähne aus ihrer Kopfbedeckung nicht ablegen lässt. Medea ist gezeichnet. Dies ist bereits der Anfangsszene immanent, von Trier eröffnet zu Medeas Verfasstheit, »daß sie, indem sie den Atem anhält, ihren Schmerz und ihren Zorn zurückhält, den gewaltigen Schmerz, den sie in ihrem Körper verspürt« (Björkman 2001: 118). Mit dem wie ein nach innen gerichteter Schrei anmutenden schockartigen Schnappen nach Luft wird diesen Emotionen ein Weg an die Oberfläche gebahnt, auch das wenig später umgesetzte Wiederauftauchen der Frau aus dem nunmehr hüfthohen Wasser »illustrates that Medea will not allow herself to be submerged and washed away permanently (or even drown herself in her distress, as some critics have it)«(Baertschi 2013: 125). »Medea steht mit ihrem nassen schwarzen Kleid regungslos im weiten flachen Meer: eine ebenso bedrohlich wie traurig wirkende Einstellung, die dadurch beendet wird, daß die Kamera in einem klatschenden Geräusch wie mit roher Gewalt nach unten gerissen wird und gurgelnd im Dunkel versinkt.« (Forst 1998: 65)

Der in die Ferne gerichtete Blick unterstreicht hierbei Medeas Wunsch, das Land zu verlassen, wie sie es der Amme später anträgt. Die Kolcherin erscheint als vom Wasser umspült, jedoch nicht bedroht - alles deutet darauf hin, dass sie durch ihren Stand im Wasser gleichermaßen Teil von und Herrscherin über diese Naturgewalt ist; die Macht des hier dunklen Wassers ist ihr immanent und wird sich sintflutartig über ihren Gegnern entladen. Medea wird zu einem Beispiel par excellence einer >mother in mourning ( $\mathrm{N}$. Loraux), einer Mutter, gefangen in einer, wie Miriam Leonard es zusammenfasst, »dramatic tension between suffering and action, mourning and revenge (Leonard 2015: 2). Nachdem Medea lange das ihr widerfahrende Unrecht hinnimmt, anstatt mit machtweibischer actio dagegen vorzugehen, entlädt sich das Angestaute schließlich, nach 
Jasons finaler Ablehnung und Verspottung ihrer Lösungsversuche und dem tätigen Zu-Boden-Schlagen seiner vormals Vertrauten, radikal an den Personen seines Begehrens. An dieser Stelle ist nun der 40-minütige Rückblick auf die Geschichte Medeas beendet und holt die filmische Gegenwart ein, die Begründungen für ihr Handeln weichen der eigentlichen Tat, eingeleitet durch das Bild der im Watt liegenden Frau, welches auch den Film eröffnet. Medea macht ihre Kinder zu Manifestationen ihres Leidens - sowohl unter den Ungerechtigkeiten des Mannes als auch unter dem letztlichen Verlust der Söhne durch ihre eigene Hand. Auf die Hinrichtung der Kinder wird im Folgenden näher eingegangen.

\section{Götter, Menschen, Ungeheuer}

Bereits in der Zwischeneinblendung der Titelgraphik »Medea « mit dem entsprechenden Filmtitel nimmt die Komposition der Körper der Söhne als stilisierte Gehängte im Namen der Mutter und die unscharfe Einblendung der schlafenden Kinder im Hintergrund zu Medeas klarer Aussage: »I long for my native land! I long for my mother, my sister, everyone ... I long for revenge«, das unausweichliche Ende der mythologischen Überlieferung und damit des Films vorweg. Die Söhne büßen beinahe biblisch für die Verfehlungen anderer (in diesem Falle Jasons), ihr Opfer befreit jedoch nicht eine Masse von Sünder:innen, sondern etabliert neuerlich die grausame Größe Medeas. Zwar kann dies als eine Art protofeministischer Akt gelesen werden, doch ist der Mord an den Kindern keinesfalls positivistisch zu sehen. Die Kinder fallen als Platzhalter dem existenziellen Konflikt der Erwachsenen, besonders der Eltern untereinander, aber eben auch der politischen Einheit des korinthischen Staates, zum Opfer und ihr Ableben befriedigt einen Rachedurst der Mutter, wie auch durch Glaukes Tod bekräftigt wird. Dennoch liegen der Hinrichtung der Söhne größere Konflikte zugrunde als die oben genannten; es stellt sich besonders die Frage nach der Bewertung der Tat vor dem Hintergrund der widersprüchlichen Natur Medeas:

[I]s she goddess or human? human or animal? masculine hero or feminine mother? civilized or savage? mad or sane? [...] the inner struggle that precedes her decision to kill her children is one of the conflicting natures, not just conflicting motives. And once that deadly resolve is realized, it is Medea's nature (not her undeniable guilt) that must be urgently clarified by the other characters in the tragedy. The question they pose in their horror and despair is not so much what kind of person could do such a thing, but, rather, is Medea a person at all, a member of humanity as a natural kind. (Daston 2002: $380 f$.

Meiner Ansicht nach sind diese Fragen hier zu einfach gestellt: Ob Medea a oder $\mathrm{b}$ ist, ist nicht eindeutig zu beantworten, vereint sie doch Charakteristika diverser mythologischer wie menschlicher Daseinsformen in sich und ist schon qua Genealogie definitiv nicht ausschließlich Göttin oder Mensch. Auch die Frage nach menschlichem gegenüber tierischem Verhalten ist zu kontextualisieren: Erkennt 
man ihre Taten als triebgesteuert an, wäre wohl Letzteres zutreffend, Affektivität hingegen würde womöglich auf Menschlichkeit - wenn auch potenziell in einem Status blinder Rache - hindeuten, wobei zu debattieren wäre, ob es sich hierbei um blinde Rache handelt oder nicht vielmehr, wie oben genannt, um kalkulierte Taten. Ebenso wäre es möglich, ihre Taten als göttlich zu bezeichnen, in Anlehnung an die vorgestellten Kategorien ihrer Genealogie. Sicherlich, göttlich im Sinne von herausragend, gut, genial wäre hier nicht gemeint, sondern vielmehr göttlich im Sinne von mächtig oder, in christlicher Tradition, auch alttestamentarisch-grausam. Die Idee, Medea könne nicht als Person anerkannt werden, erscheint hingegen unzulänglich, wird sie doch durch Kreon als Gefahr für Staat und Leben gesehen und als natürliche Person vor dem Recht des Staates (dessen ausführende Gewalt er im Ausspruch des Bannes über die Kolcherin ist) zum Verlassen des Staatsgebietes verurteilt.

Wo Evokationen von äußeren Ähnlichkeiten zu Kreaturen wie Selkies, Meerjungfrauen oder Meerhexen passend sind und bereits Euripides, auf dessen Tragödie von Triers Umsetzung ja basiert, das Meer bzw. das Wasser als Element Medeas anerkennt (vgl. Joseph/Johnson 2008), wird die Ungeheuerlichkeit Medeas auch durch filmische Spezifika unterstrichen. Nicht nur wird die Kamera zu Beginn von den Medea umgebenden dunklen Wassermassen überspült, was Beunruhigung und Düsternis unterstreicht, auch dominieren Normalsicht und Aufsicht zur Figur. Es schwankt der

point of view [...] zwischen der menschlichen und der göttlichen Perspektive. Der Mensch ist hin- und hergerissen zwischen Autonomie auf der einen und Passivität und Ohnmacht auf der anderen Seite, was wiederum die Ambivalenz der Figur, zum Beispiel Medeas Doppelrolle als Täterin und Opfer, untermalt. (Schorr 2009: 16)

Bevor Medea überspült wird, wird die auch hier eingeführte >Gotthaftigkeit< (auch hier: alttestamentarischer, zürnender Gott) ad extremum geführt, wenn im Hintergrund die einrollenden Wellen von erweiterten Quarten (augmented fourths) begleitet werden - »in Western music from Baroque cantatas to Disney cartoons, a time-worn metaphor for the devil« (Joseph/Johnson 2008: 118). Ob hier nun Medea oder aber die sinnbildliche Flut teuflisch ist, bleibt unklar.

The modern Medea is at once unnatural and all too natural. In her, the orders of specific natures and universal Nature collide, turning her into a moral paradox. [...] infanticide committed by the mother eludes ethical analysis. Instead, ingenuity is channeled into imagining circumstances so horrendous that a mother might kill her children in the service rather than in violation of maternal nature. (Daston 2002: 385)

Liest man Medea entsprechend unabhängig von den oben genannten Kategorien primär als Mutter, ergeben sich weitere Paradoxien in der Evaluation ihrer Tat. Sie umsorgt die Kinder, so bspw. als der Jüngere fällt und sein Knie aufschlägt, hält bei der Durchführung ihrer Tat leidend inne, hebt gar den älteren Sohn hoch, bis ihre Kräfte sie verlassen, um seinen Tod offenbar doch noch hinauszu- 
zögern. Doch kündigt sich bereits früh, im Wutausbruch gegenüber der Amme, die bevorstehende Grausamkeit an. Bereits als die Kinder schlafen, überschattet Medeas Silhouette die Lagerstatt - Müller assoziiert dies mit der abstrakten Darstellung der Mutter als Todesengel (vgl. Müller 2000: 160), wiederum eine Übermenschlichkeit attestierend. Vollendet wird Medeas Tat hingegen nicht in Dunkelheit oder Zwielicht, sondern bei strahlendem Sonnenschein, nahezu vor den Augen des Großvaters Helios. Das Licht erhellt nach und nach das Gräsermeer, bevor es auch den Galgenbaum umfasst und Medea in das Gesicht scheint. Die Sommerwiese, in deren Mitte erhöht der Galgenbaum thront, erscheint hier in goldbraunen Farben, die Textur erinnert während der Kamerafahrt über die Ebene an Wellen auf dem Meer, da sich auch die Gräser vom Wind bewegt wellenförmig regen. Medea erscheint zuletzt still, fast gelähmt, wohl ob ihrer Tat, hingegen ist sie es, die sich im wahrsten Sinne zu neuen Ufern begeben kann. Im scharfen Gegensatz hierzu steht Jason, der am Schluss im Zentrum des Kaders inmitten des Gräsermeeres zusammensinkt und in einer Reihe von Fade-outs mit selbigem verschmilzt und zu ertrinken scheint.

\section{FAZIT}

Lars von Triers Medea stellt nicht per se das Meer als transkulturellen Erinnerungsraum aus, sondern etabliert die Hauptfigur, die gleich zu Beginn des Filmes den Fluten entsteigt und fortwährend mit Wasser, Wellen und nicht zuletzt auch dem Meer in symbolischer wie spatialer Weise eng verbunden ist, als kulturellen Gedächtnisort, den es auf der Rezeptionsseite durch individuelle Interpretations- und Gedächtnisleistung zu entschlüsseln gilt. Medea stellt nicht nur eine androgyne und damit geschlechtlich ambivalente Figur dar, die somit in sich die divergierende kulturelle Codierung von Wellen und Wasser vereint. Durch das Mythologem ihres griechisch-römischen Namens und die Verortung in einer nordisch-mythischen Vorzeitlichkeit werden zwei Mythostraditionen einander zugeführt, die jeweils das kulturelle Gedächtnis einer westlichen Kulturgemeinschaft prägen und durch ihre Juxtaposition Medea selbst als einen lieu de mémoire divisé etablieren. Sie wird zu einem middle ground unterschiedlicher Kulturtraditionen, Wertekanons und Diskurse. Medea stellt eine Arbeit am klassischen Mythos dar, die ausgesprochen transkulturell zu lesen ist und zeitgleich als Echokammer, Referenzraum und Erinnerungsort angesehen werden kann, welche die Rezipient:innen aufgrund ihrer individuellen Zugriffe auf kulturelle Kanones, textuelle Elemente des eigenen kulturellen Gedächtnisses sowie visuelle Traditionen kinematographischer Stilrichtungen prozesshaft bedeutungstragend machen. 


\section{LITERATUR}

Asbjørn Jøn, Allan (1998): Dugongs and Mermaids, Selkies and Seals. In: Australian Folklore 13, S. 94-98.

Assmann, Jan (1988): Kollektives Gedächtnis und kulturelle Identität. In: Ders./Tonio Hölscher (Hg.): Kultur und Gedächtnis. Frankfurt a.M., S. 9-19.

Baertschi, Annette M. (2013): Rebel and Martyr: The Medea of Lars von Trier. In: Konstantinos P. Nikoloutsos (Hg.): Ancient Greek Women in Film. Oxford, S. 117-136.

Björkman, Stig (2001): Trier über von Trier. Gespräche mit Stig Björkman. Hamburg.

Borschberg, Peter/North, Michael (2010): Transcending Borders: The Sea as Realm of Memory. In: Asia Europe Journal 8, S. 279-292.

Christie, Ian (2000): Between Magic and Realism: Medea on Film. In: Edith Hall/Fiona Macintosh / Oliver Taplin (Hg.): Medea in Performance 1500-2000. Oxford, S. 144165.

Daston, Lorraine (2002): The Morality of Natural Orders: The Power of Medea. Vortrag in der Reihe The Tanner Lectures on Human Values, gehalten am 6. November 2002 an der Harvard University, S. 373-411; online unter: https://tannerlectures.utah. edu/_documents/a-to-z/d/daston_2002.pdf [Stand: 1.10.2020].

Deleuze, Gilles/Guattari, Félix (2006): 1440 - Das Glatte und das Gekerbte. In: Jörg Dünne/Stephan Günzel (Hg.): Raumtheorie. Grundlagentexte aus Philosophie und Kulturwissenschaften. Frankfurt a.M., S. 434-448.

Eason, Cassandra (2008): Fabulous Creatures, Mythical Monsters and Animal Power Symbols. Greenwood.

Forst, Achim (1998): Breaking the Dreams. Das Kino des Lars von Trier. Marburg.

Helmreich, Stefan (2017): The Genders of Waves. In: Women's Studies Quarterly 45, H. 1/2, S. 29-51.

Joseph, Susan/Johnson, Marguerite (2008): "An Orchid in the Land of Technology": Narrative and Representation in Lars von Trier's Medea. In: Arethusa 41, H. 1, S. 113-132.

Kristeva, Julia (1982): Powers of Horror. An Essay on Abjection. Aus dem Franz. v. Leon S. Roudiez. New York.

Leonard, Miriam (2015): „I know what has to happen «: Tragedy in Lars von Trier's Medea. In: Theory and Event 18, H. 2, o.S.

Lütkehaus, Ludger (Hg.; 2001): Mythos Medea. Texte von Euripides bis Christa Wolf. Stuttgart.

Müller, Marion (2000): Vexierbilder. Die Filmwelten des Lars von Trier. St. Augustin.

Schorr, Andrea (2009): Frauenfiguren in den Filmen Lars von Triers. Von Opfern und Tätern. Saarbrücken. 
